Research Article

\title{
A Novel Ultrawideband Gear-Shaped Dielectric Ring Resonator Antenna
}

\author{
Mehdi Ghorbani (D) and Habib Ghorbaninejad \\ Department of Electrical Engineering, University of Guilan, Rasht, Iran \\ Correspondence should be addressed to Mehdi Ghorbani; ghorbani.me@gmail.com
}

Received 20 May 2021; Accepted 29 June 2021; Published 13 July 2021

Academic Editor: Noorbakhsh Amiri Golilarz

Copyright (c) 2021 Mehdi Ghorbani and Habib Ghorbaninejad. This is an open access article distributed under the Creative Commons Attribution License, which permits unrestricted use, distribution, and reproduction in any medium, provided the original work is properly cited.

In this study, a novel ultrawideband (UWB) dielectric ring resonator (DRR) antenna has been proposed. DRR antennas include a single monopole antenna in the center of a ground plane and a dielectric with a symmetric structure around the monopole. This structure will lead to ultrawide band antenna. However, it is still possible to enhance the antenna bandwidth. In this study, we combine the DRR structure with an array antenna. The proposed antenna includes a circular array of four triangle resonators, which is rotated around the center of the triangle base to form a gear-shaped ring resonator antenna. In this design, characteristics of all these antennas are combined to enhance the antenna bandwidth including triangular dielectric resonator, circular array antenna, dielectric ring resonator structure, and a quarter-wave electric monopole. Triangular dielectric resonator antennas are wideband and in small size. Ring resonator antennas are inherently ultrawideband. Quarter-wave electric monopole and circular array structure can also enhance antenna bandwidth. This novel shape of the DRR antenna possesses the wider impedance bandwidth compared to similar works. Impedance bandwidth is $150 \%(5.2-36.1 \mathrm{GHz})$, and the bandwidth ratio is $1: 6.9$, which is much greater than earlier reports.

\section{Introduction}

Dielectric resonator antennas (DRA) are very attractive topics due to a great variety of reasons. These antennas' benefits include small size, low cost, high radiation efficiency, and low losses due to the elimination of the conductor loss. Moreover, UWB antennas can widely be used in portable broadband wireless, radar, navigation, GPS, the biomedical system, mobile satellite communications, the direct broadcast system, and telemetry. For wideband applications, a variety of techniques were applied to improve the bandwidth of these antennas. Dielectric ring resonator (DRR) antennas are a kind of DRAs which intrinsically possess wide bandwidth.

In 2002, a research group discovered that the combination of a quarter-wave electric monopole and a ring resonator will lead to wideband structure [1]. Hemispherical, conical, and annular resonators are the most common shapes for design of DRR antenna. In [2], a hemispherical and a conical DRR antenna was investigated and the bandwidth ratio of $4: 1$ was reported. In [3], an annular DRR with the step in outer radius was announced.

In DRR antennas, as will be discussed in more detail in Section 4 , the first resonance is due to the monopole in $\lambda / 4$, and the next resonance is due to the resonator. In fact, the second resonance is activated by mode TM01 $\delta$ [4]. There are series of resonances in odd multiples of quarter wavelength. For achieving high bandwidth, plenty of research has been driven toward feed modification and resonator structure. As an illustration, stacked forms of aforementioned shapes can significantly improve impedance bandwidth. A pawn-shaped DRR antenna was designed by attaching conical and hemispherical shapes [5]. In [6], two stacked conical DRR with the same and opposite directions were investigated. In [7], a stacked form of a double annular DRR antenna with different permitivities was investigated. In [8], three different methods of impedance matching, dielectric, and ground plane are applied in order to enhance the antenna 
bandwidth. As mentioned before, modification of the feeding is another technique on impedance matching and bandwidth enhancement. Other shapes of feeding include L-shaped microstrip [9], annular-shaped microstrip [10], a tilted modified square-shaped aperture [11], and circularshaped offset feed [12] for 3-band applications. In [13], the high permittivity and low losses properties of the ceramic material were used for manufacturing the magnetic resonance probes.

In recent studies, more focus is on the feed modification methods to improve antenna features. However, beside this, it is still possible to improve the antenna characteristics using traditional feeding for new structures. In this study, the potential of combination of the DRR antenna and array antenna is demonstrated.

Referenced studies reveal inherent potential of DRR antennas for wideband applications. However, it is possible to improve antenna bandwidth more efficiently. In this regard, based on our knowledge, we have combined and promoted array formation of elements on dielectric ring resonator antenna concept for the first time. Array formation is usually used to enhance antenna gain. However, it can be used to enhance antenna impedance bandwidth. Using these two techniques has concurrently enhanced antenna impedance bandwidth significantly. The impedance bandwidth of the antenna has been outlined and compared with some works of literature in Table 1 . This table clearly demonstrates the efficacy of the design. For antenna elements, an isosceles triangular dielectric resonator (TDR) has been opted due to its potential for wideband applications [14].

In this study, a novel shape of ultrawideband DRR antenna has been proposed. The DRR antenna is a gearshaped DRR antenna, including a circular array of four triangular dielectric resonators. The resonator is located on an extended conducting ground plane and is fed by a quarter-wave monopole probe. The proposed antenna has been simulated with HFSS, and dimensions have been optimized using an extensive parametric study in order to achieve best results.

In this study, in Section 2, antenna configuration will be discussed. In Section 3 (design steps), the effect of antenna parameters on the reflection coefficient chart will be shown and explained. Then, the antenna will be simulated with different softwares and the result will be compared with relevant works in Section 4. In Section 5, theory of the antenna will be elaborated. Finally in Section 6, the whole work will be concluded.

\section{Antenna Configuration}

Figure 1(a) shows the 3D view of the proposed antenna. The resonator is located on the conducting ground plane and is excited with a coaxial probe as a quarter-wave monopole. The ground plane is square shaped with dimensions of $g \times g$ $\left(70 \times 70 \mathrm{~mm}^{2}\right)$. The probe is a $50 \mathrm{ohm}$ coaxial cable with an inner conductor radius of $0.65 \mathrm{~mm}$ and height of $p$.

The resonator includes the dielectric material of RO3010 with permittivity of 10.2 and dissipation factor 0.0035 .
Figures $1(\mathrm{~b})$ and $1(\mathrm{c})$ show the DRR configuration and antenna parameters. The proposed DRR is composed of four arrays of triangle elements, which are rotated around the center of the base side of the triangle (center of the coordinate system, and dimensions of these triangles consist the base side $(b)$, the median $(a)$, and central hole radius $(r)$ ). Table 2 provides the final dimensions of the proposed antenna.

\section{Design Steps}

Step 1. Triangle median size (a): Figure 2 shows the antenna reflection coefficient in accordance with the triangle median (a).

The triangle antenna's median is one of the most important parameters in impedance bandwidth. In the first half of the bandwidth, there are two resonances due to monopole (at $6.2 \mathrm{GHz}$ and $15.1 \mathrm{GHz}$ ) and two other resonances due to the resonator (at $9.8 \mathrm{GHz}$ and $11.8 \mathrm{GHz}$ ). Lower values of the triangle median deteriorate the reflection coefficient at the frequencies around $13 \mathrm{GHz}$, and its higher values worsen the reflection coefficient at the frequencies around $8 \mathrm{GHz}$. Therefore, $a=6.0 \mathrm{~mm}$ is the best value for triangle median.

Step 2. Triangle base size (b): Figure 3 shows the reflection coefficient of the proposed antenna in accordance with the base size of the triangle $(b)$. This figure shows that this parameter does not have a significant effect on the first half of the bandwidth. Also, it shows that the decrease of base size slightly improves antenna bandwidth, while disturbing return loss at some other frequencies including 8,20, and $32 \mathrm{GHz}$. Moreover, it moves resonant frequencies in the second half of the bandwidth. On the whole, $b=2.0 \mathrm{~mm}$ is the best value for the size of the triangle base.

Step 3. Central hole radius $(r)$ : at the next step of the design process, the effect of central hole radius of the DRR is investigated. Figure 4 shows the reflection coefficient of the proposed antenna with the central hole radius $(r)$. This figure depicts that an increase in $r$ improves bandwidth, but it increases return loss. Therefore, it disturbs the return loss in some frequencies. Also, it can be seen that the parameter of $r$ does not have a significant effect on the resonant frequencies.

Step 4. DRR antenna height (h): at the next step of the design process, the proposed DRR antenna height is investigated. Figure 5 shows the reflection coefficient of the proposed antenna in accordance with the parameter of $h$. This figure shows that an increase in the parameter of $h$ reduces impedance bandwidth and simultaneously disturbs return loss in some ranges of frequencies, especially around $13.5 \mathrm{GHz}$ and $21 \mathrm{GHz}$. For lower values of $h$, return loss is improved at higher frequencies, in order to improve bandwidth but it disturbs it at lower frequencies, especially around $25 \mathrm{GHz}$ and $33 \mathrm{GHz}$. Therefore, it restricts the impedance bandwidth. From the figure, $h=7.0 \mathrm{~mm}$ is the best value for the antenna height. 
TABLE 1: Comparison between the proposed antenna and some previous literatures.

\begin{tabular}{lcccc}
\hline Reference no. & Frequency range $(\mathrm{GHz})$ & Bandwidth $(\%)$ & Bandwidth ratio & Permittivity \\
\hline 7 & $5.6-22.4$ & 126 & $4: 1$ & $3.6: 1$ \\
8 & $1.95-7.05$ & 110 & $4.2: 1$ & 10 \\
10 & $5.5-23.0$ & 122 & $5.4: 1$ & 10 \\
11 & $2.8-15.2$ & 138 & $1.5: 1$ & $3.8: 1$ \\
12 & $3.6-5.5$ & 42 & $2.1: 1$ & 10 \\
13 & $1.8-6.9$ & 117 & $6.9: 1$ & 22 \\
15 & $3.49-7.20$ & 66.7 & 150 & 10.2 \\
PA $^{*}$ & $5.2-36.1$ & 150 & \\
\hline
\end{tabular}

${ }^{*} \mathrm{PA}$, the proposed antenna.

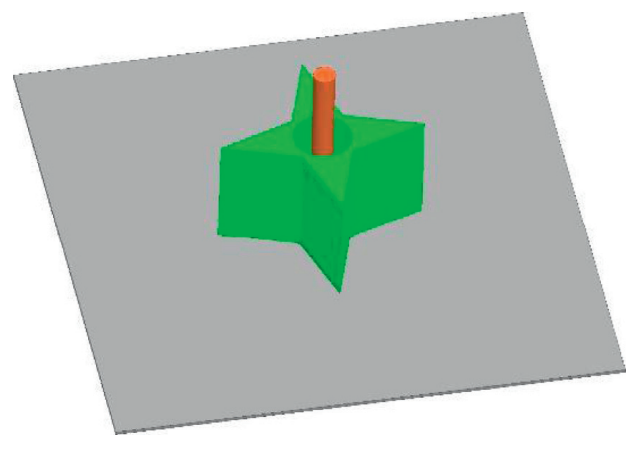

(a)

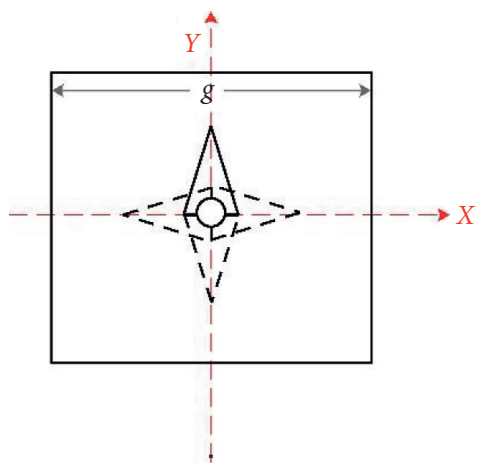

(b)

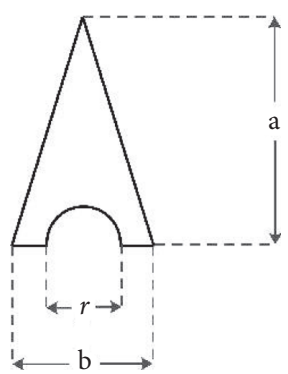

(c)

FIgure 1: (a) 3D view, (b) DRR configuration, and (c) elements of the proposed DRR.

TABLE 2: Final dimensions of the proposed antenna (all dimensions in $\mathrm{mm}$ ).

\begin{tabular}{lcc}
\hline Parameter & Description & Value $(\mathrm{mm})$ \\
\hline$g$ & Ground plane width and length & 70.0 \\
$a$ & Triangle median & 6.0 \\
$b$ & Triangle base & 2.0 \\
$r$ & Drilled hole diameter & 1.7 \\
$h$ & DRR antenna height & 7.0 \\
$p$ & Probe height & 12.0 \\
$e$ & Probe radius & 0.65 \\
\hline
\end{tabular}

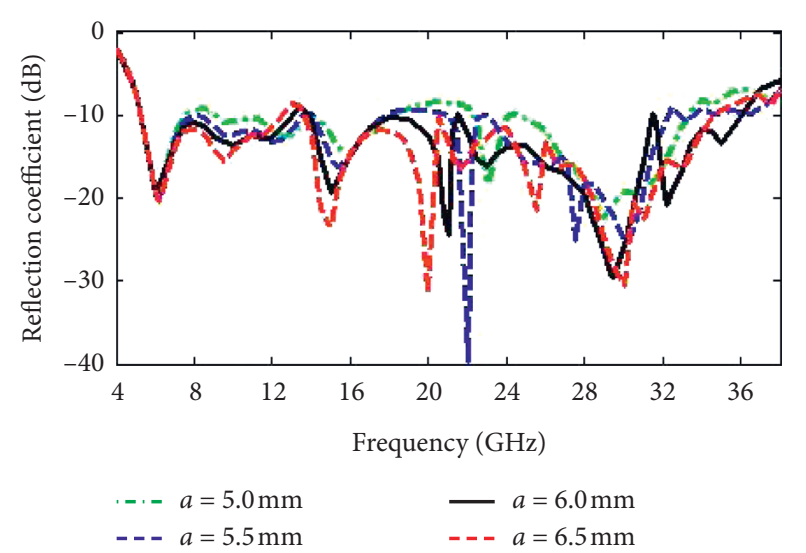

FIGURE 2: Reflection coefficient of the proposed antenna in accordance with triangle median.

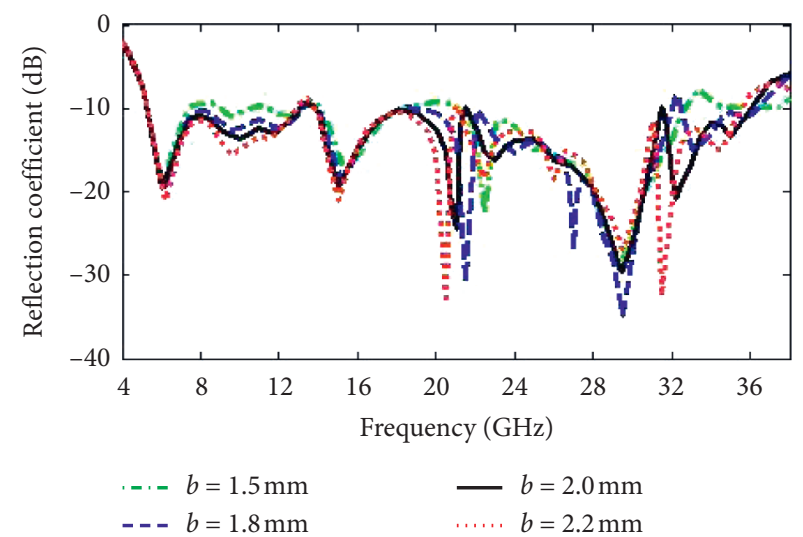

FIGURE 3: Reflection coefficient of the proposed antenna in accordance with base triangle.

Step 5. Probe height $(p)$ : Figure 6 shows the effect of the probe height on antenna return loss. This figure depicts that probe height has a significant effect in almost all resonance frequencies and the reflection coefficient chart. From this figure, it can clearly be seen that the probe height of $p=12 \mathrm{~mm}$ possesses the best reflection coefficient chart and impedance matching feature.

Step 6. Probe radius (e): Figure 7 shows the effect of the probe diameter on the antenna reflection coefficient. From 


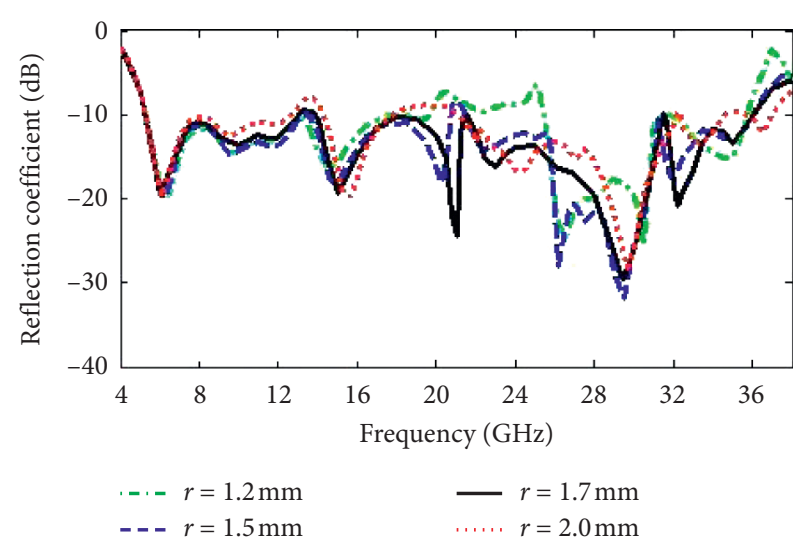

FIGURE 4: Reflection coefficient of the proposed antenna in accordance with central hole radius.

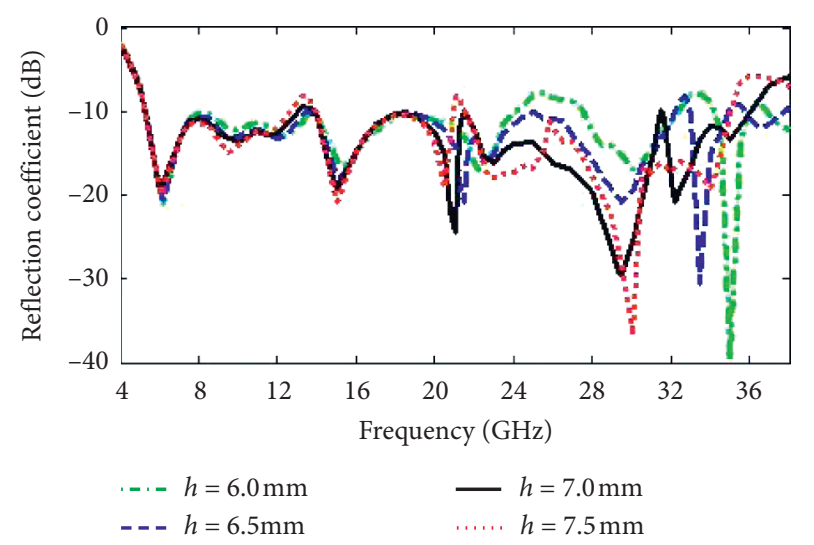

FIGURE 5: Reflection coefficient of the proposed antenna in accordance with DRR antenna height.

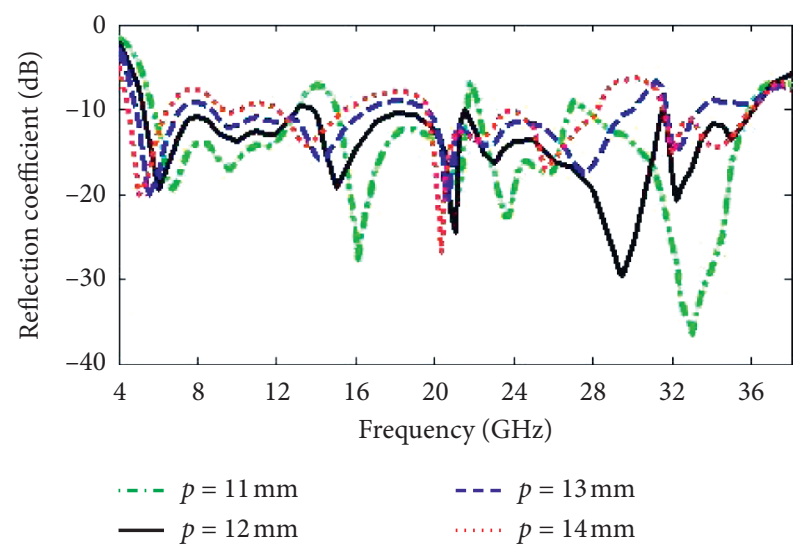

FIGURE 6: Reflection coefficient of the proposed antenna in accordance with probe height.

this figure, it can clearly be seen that the probe diameter has no effect on the antenna bandwidth in lower values of $e$.

For all of the values of $e$, starting and ending points are coincided; however, in some higher frequency ranges, they trespass the criteria of $-10 \mathrm{~dB}$ of the reflection coefficient chart. But this issue can be solved by other parameters

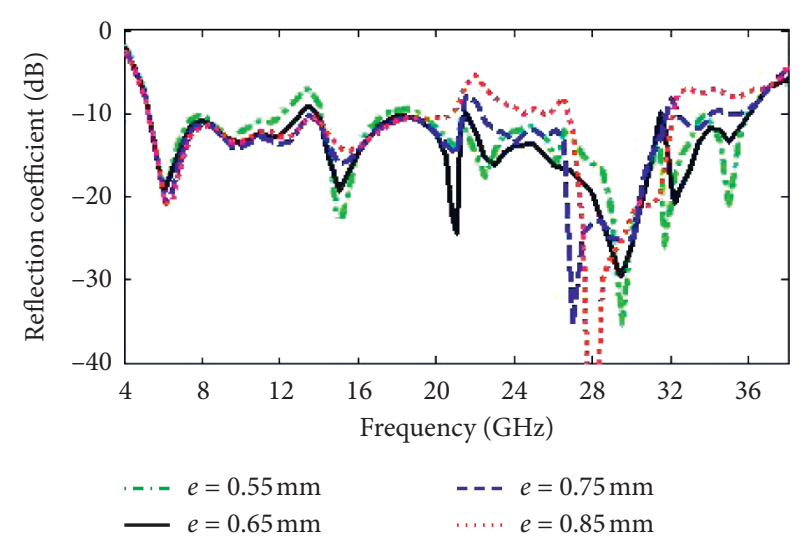

Figure 7: Reflection coefficient of the proposed antenna in accordance with probe diameter.

reoptimization. It can also be seen that almost all frequency resonances remain constant.

\section{Results and Comparison}

The proposed antenna has been simulated and optimized using HFSS, and a good characteristic has been achieved. The impedance bandwidth of the antenna is $150 \%$ $(5.2-36.1 \mathrm{GHz})$. Figure 8 shows the efficiency and the gain of the proposed antenna. Efficiency of the proposed antenna is between $93 \%$ and $97 \%$ in the whole bandwidth.

Table 1 provides the summary comparison between the proposed antenna and some similar studies. From the table, it can be found that the proposed antenna possesses an excellent ultrabandwidth feature. The radiation pattern is also good in lower frequencies, but it disturbed slightly in higher frequencies.

4.1. Comparison of the Results from HFSS and CST. The proposed antenna has been simulated with HFSS and CST, and the same results have been achieved. Figure 9 shows the reflection coefficient of the antenna simulated with these two softwares, which are based on the method of moment $(\mathrm{MoM})$ and finite element method (FEM), respectively. It can clearly be seen that the same results have been achieved. Results from these two different methods validate the antenna characteristics.

Figure 10 depicts the radiation pattern of the antenna at $10 \mathrm{GHz}, 20 \mathrm{GHz}$, and $30 \mathrm{GHz}$ simulated with HFSS and CST. It can clearly be seen that a good agreement has been achieved.

Figure 11 shows the E-field patterns of the proposed antenna at resonance frequencies. Figure 11 (a) $(6.2 \mathrm{GHz})$ shows the main resonance of the monopole at $6.2 \mathrm{GHz}(\lambda / 4)$. At this frequency TE10 has been activated. Similarly, Figure 11(d) depicts the second resonance of monopole, and the TE mode is activated.

In Figures 11(b), 11(c), and 11(e), electric field distributions inside the DRR have been shown. These figures demonstrate that $\mathrm{Ez} \neq 0$ and $\mathrm{Hz}=0$. Therefore, $\mathrm{TM}$ modes are activated due to resonator overall dimension and configuration. 


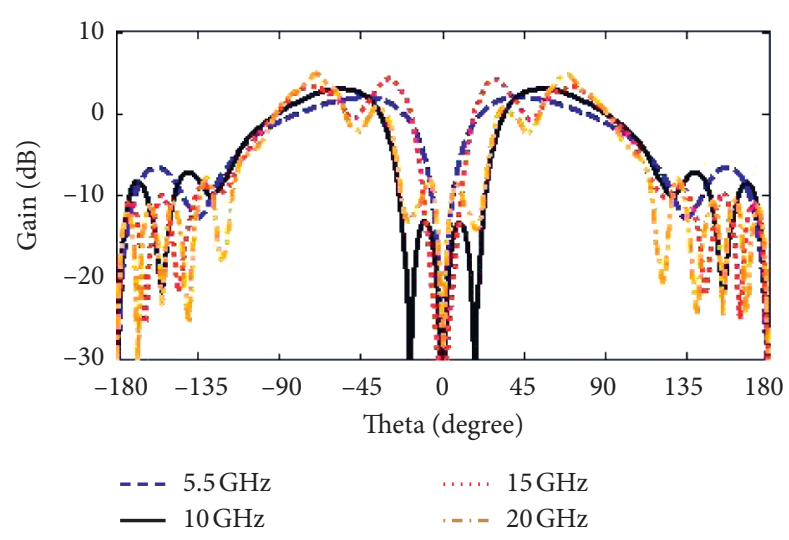

(a)

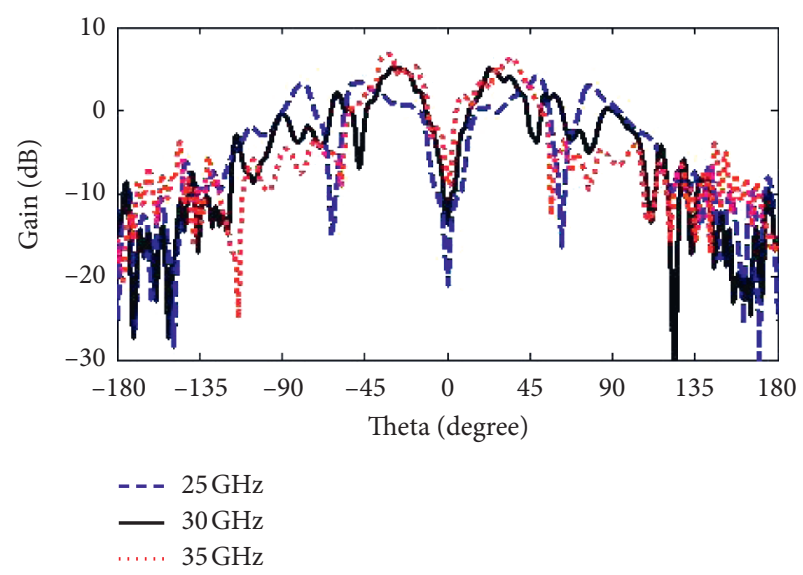

(b)

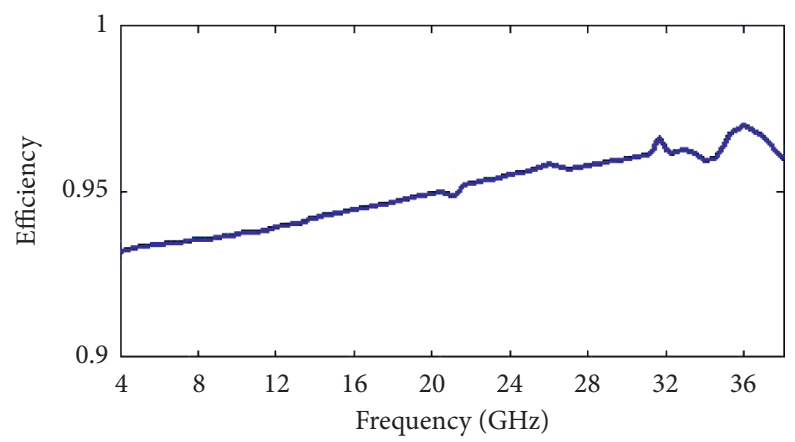

(c)

Figure 8: (a), (b) Gain of the proposed antenna. (c) Efficiency.

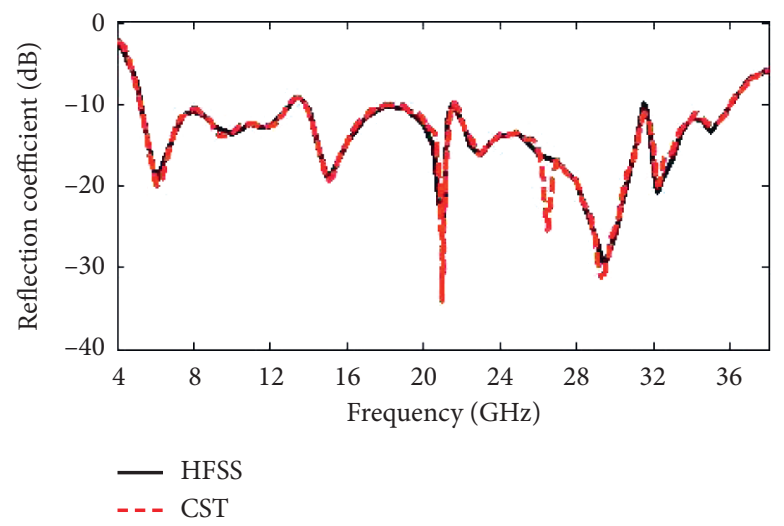

FIGURE 9: Reflection coefficient of the proposed antenna simulated with HFSS and CST.

Figure $11(\mathrm{~g})(29.5 \mathrm{GHz})$ is due to electric monopole (at $5 \lambda / 4$ ), and Figures 11(f) and 11(h) depict the electric field distributions produced by the resonator. These frequencies include both $\mathrm{Ez}$ and $\mathrm{Hz}$, and hybrid modes have been activated.

\section{Theory}

The monopole has resonant frequencies in odd multiples of quarter wavelength. In this case, monopole height is $p=12 \mathrm{~mm}$. Therefore, according to equation (1), the resonant frequencies of the monopole are $6.25 \mathrm{GHz}$, $18.75 \mathrm{GHz}$, and $31.25 \mathrm{GHz}$.

$$
f_{r}=\left\{\frac{1}{4}, \frac{3}{4}, \frac{5}{4}, \ldots\right\} *\left(\frac{C}{P}\right)
$$

where $C$ is the light velocity in free space. These resonant frequencies are shown in Figure 12(a), red dash line. In Figure 12(a), the reflection coefficient of the antenna without gear resonator has been shown. This figure shows that the antenna resonates at $5.80 \mathrm{GHz}, 17.80 \mathrm{GHz}$, and $30.2 \mathrm{GHz}$, which are close to the resonance frequencies calculated from 


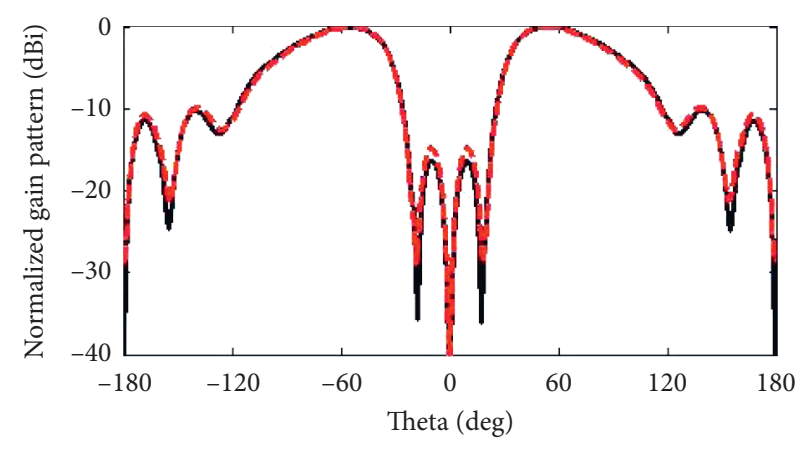

$\begin{array}{ll}\text { - } & \text { HFSS } \\ -- \text { CST }\end{array}$

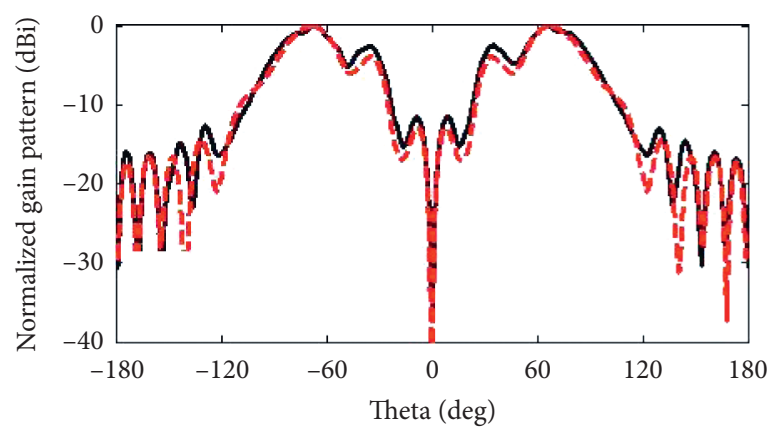

- HFSS

(a)

(b)

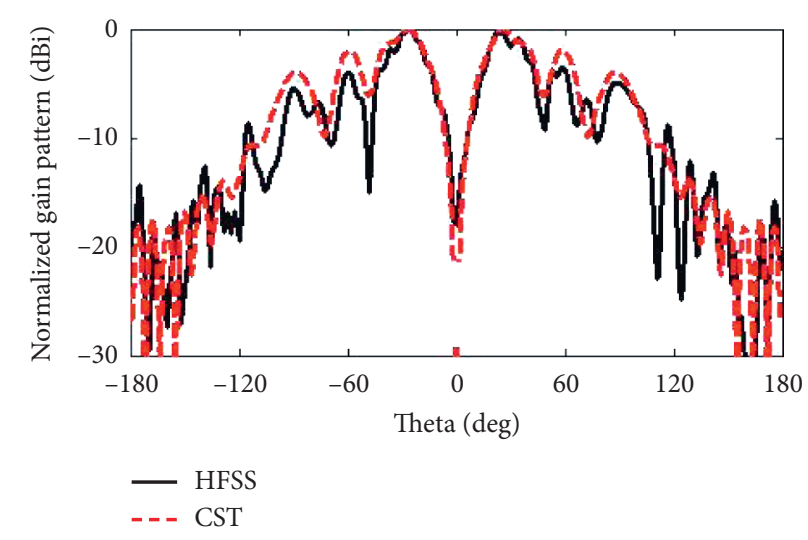

(c)

FIGURE 10: Radiation pattern of the proposed antenna simulated in HFSS and CST at (a) $10 \mathrm{GHz}$, (b) $20 \mathrm{GHz}$, and (c) $30 \mathrm{GHz}$.

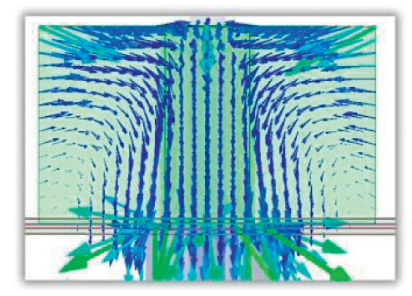

(a)

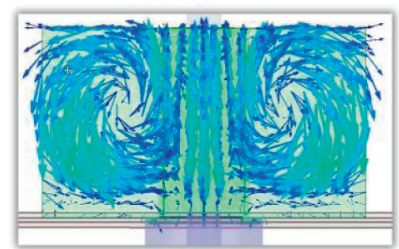

(e)

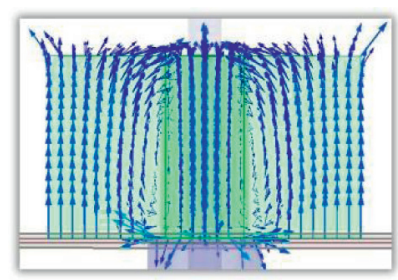

(b)

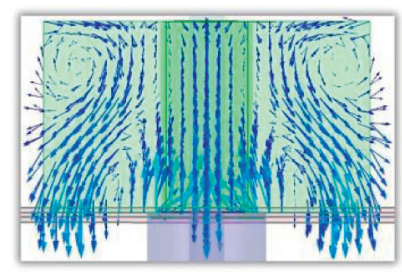

(f)

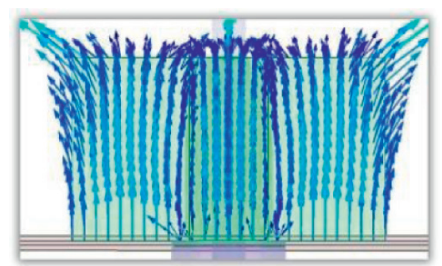

(c)

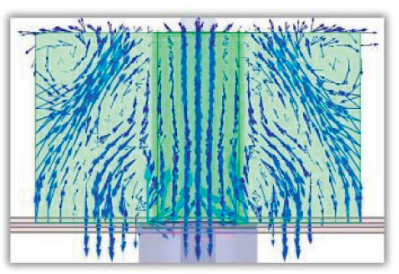

(g)

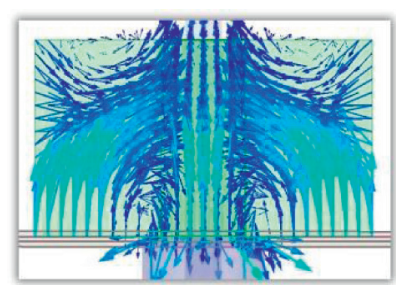

(d)

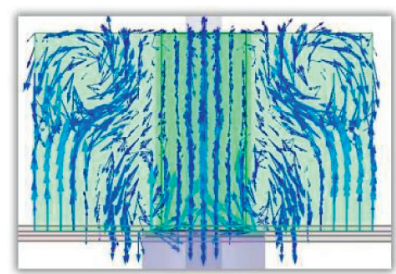

(h)

FIGURE 11: E-field pattern of the antenna at (a) $6.2 \mathrm{GHz}$, (b) $9.8 \mathrm{GHz}$, (c) $11.8 \mathrm{GHz}$, (d) $15.1 \mathrm{GHz}$, (e) $21 \mathrm{GHz}$, (f) $22.8 \mathrm{GHz}$, (g) $29.5 \mathrm{GHz}$, and (h) $32.2 \mathrm{GHz}$. 

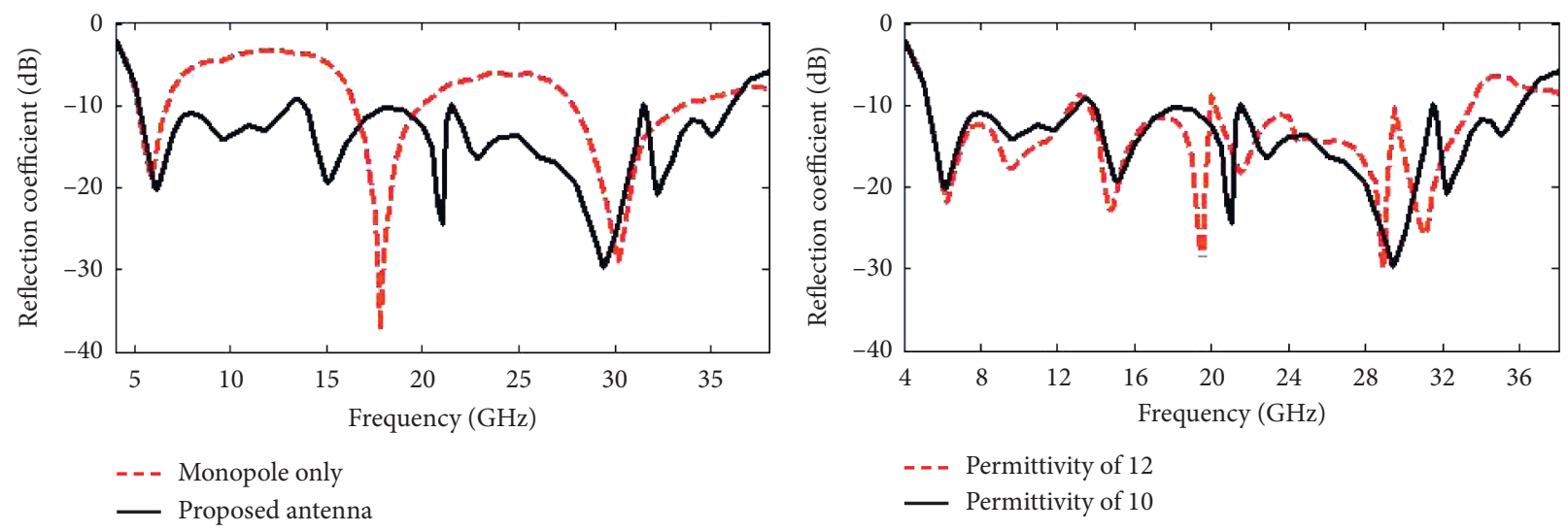

(a)

(b)

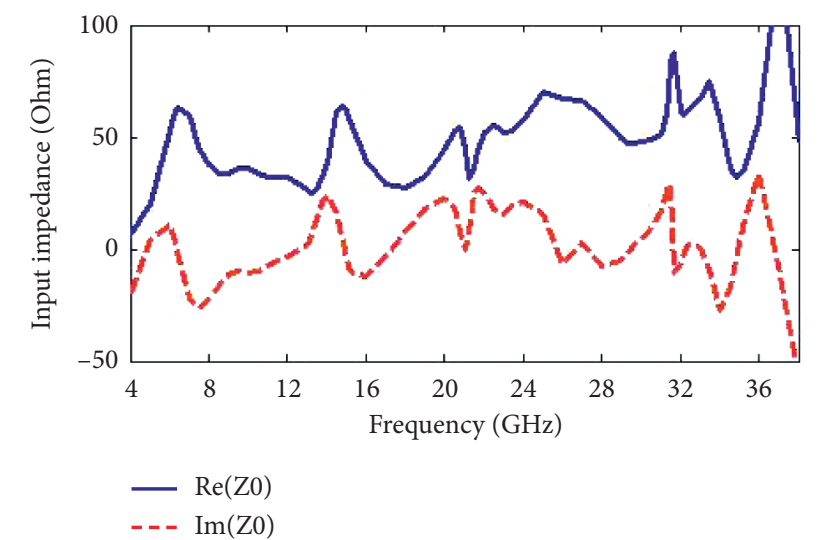

(c)

FIGURE 12: (a) Reflection coefficient of the proposed antenna compared to monopole alone. (b) Reflection coefficient for permittivity of 10.2 and 12. (c) Real and imaginary part of the input impedance.

the equation. These frequencies moved to $6.20 \mathrm{GHz}$, $15.10 \mathrm{GHz}$, and $29.5 \mathrm{GHz}$ due to applying the resonator. These reductions, particularly at the second resonance, are from capacitive loading of the resonator. Therefore, these frequencies are expected to remain unchanged by changing the relative permittivity of the resonator. Figure 12(b) shows that these resonance frequencies remain unchanged with relative permittivity, while other resonance frequencies have been decreased with increasing the permittivity of the resonator.

Figure 12(c) shows the real and imaginary part of the characteristic impedance. This figure shows that the imaginary part of the $Z_{0}$ at $6.20 \mathrm{GHz}, 29.5 \mathrm{GHz}$ are positive, and then, they have not significantly moved after applying the resonator, while it is negative at $15.1 \mathrm{GHz}$. Therefore, this resonance frequency has been decreased $3.65 \mathrm{GHz}$, in comparison with the monopole only.

\section{Conclusion}

In this study, a novel ultrawideband DRR antenna has been proposed. It includes a quarter-wave monopole which resonates in odd multiples of quarter wavelength. Therefore, using a wideband structure can cover these gaps between monopole resonances to enhance antenna bandwidth. Moreover, it is well known that array antennas are used in wideband applications. Therefore, combination of these two techniques can enhance the impedance bandwidth. The last step is to find a suitable shape for array elements. In this regard, a triangular dielectric resonator (TDR) is opted. TDR antennas are small sized and wideband. In general, designing an antenna using all mentioned above has the potential of wideband application.

The proposed antenna consists of a novel gear-shaped DRR which is located on the conducting ground plane and is fed using a coaxial probe as a quarter-wave monopole. The DRR profile consists of a circular array of four triangle resonators. The resonators turn around the monopole and connect to each other from the apex of the base side to form a gear shape. The proposed antenna is designed and optimized using HFSS, and an ultrahigh bandwidth has been achieved. The proposed antenna has an impedance bandwidth of $150 \%(5.2-36.1 \mathrm{GHz})$ with $6.94: 1$ bandwidth ratio.

\section{Data Availability}

All the data used to support the findings of this study are included within the article. 


\section{Conflicts of Interest}

The authors declare that they have no conflicts of interest.

\section{References}

[1] A. Ittipiboon, A. Petosa, and S. Thirakoune, "Bandwidth enhancement of a monopole using dielectric antenna resonator loading," in Proceedings of the ANTEM Conference, pp. 387-390, Montreal, Canada, August 2002.

[2] D. Guha, B. Gupta, and Y. M. M. Antar, "Hybrid monopoleDRAs using hemispherical/conical-shaped dielectric ring resonators: improved ultrawideband designs," IEEE Transactions on Antennas and Propagation, vol. 60, no. 1, 2012.

[3] C. Ozzaim, "Monopole antenna loaded by a stepped-radius dielectric ring resonator for ultrawide bandwidth," IEEE Antennas and Wireless Propagation Letters, vol. 10, 2011.

[4] D. Guha, Y. M. M. Antar, A. Ittipiboon, A. Petosa, and D. Lee, "Improved design guidelines for the ultra wideband monopole-dielectric resonator antenna," IEEE Antennas and Wireless Propagation Letters, vol. 5, pp. 373-376, 2006.

[5] D. Guha, B. Gupta, and Y. M. M. Antar, "New pawn-shaped dielectric ring resonator loaded hybrid monopole antenna for improved ultrawide bandwidth," IEEE Antennas and Wireless Propagation Letters, vol. 8, 2009.

[6] C. Ozzaim, F. Üstüner, and N. Tarim, "Stacked conical ring dielectric resonator antenna excited by a monopole for improved ultrawide bandwidth," IEEE Transactions on Antennas and Propagation, vol. 61, no. 3, 2013.

[7] Y. X. Guo, Y. F. Ruan, and X. Q. Shi, "Wide-band stacked double annular-ring dielectric resonator antenna at the endfire mode operation," IEEE Transactions on Antennas and Propagation, vol. 53, no. 10, 2005.

[8] M. N. Jazi and T. A. Denidni, "Design and implementation of an ultrawideband hybrid skirt monopole dielectric resonator antenna," IEEE Antennas and Wireless Propagation Letters, vol. 7, 2008.

[9] W. Chang and Z. Feng, "Investigation of a novel wideband feeding technique for dielectric ring resonator antennas," IEEE Antennas and Wireless Propagation Letters, vol. 8, 2009.

[10] R. K. Chaudhary, R. Kumar, and K. V. Srivastava, "Wideband ring dielectric resonator antenna with annular-shaped microstrip feed," IEEE Antennas and Wireless Propagation Letters, vol. 12, pp. 595-598, 2013.

[11] D. Pathak, S. K. Sharma, and V. S. Kushwah, "Investigation on circularly polarized ring dielectric resonator antenna for dualband wireless applications," Progress In Electromagnetics Research M, vol. 62, pp. 123-130, 2017.

[12] K. Tulasi, K. Jyothi, B. L. V. Kumari et al., "A novel offset feed annular ring dielectric resonator antenna for bandwidth enhancement," International Journal of Engineering and Advanced Technology, vol. 94 pages, 2020.

[13] M. Moussu, R. Abdeddaim, M. Dubois et al., "A semi-analytical model of high permittivity dielectric ring resonators for magnetic resonance imaging," IEEE Transactions on Antennas and Propagation, vol. 68, no. 8, 2020.

[14] M. Ghorbani, M. Khalily, H. Ghorbaninejad, P. Xiao, and R. Tafazolli, "Bandwidth enhancement and radiation characteristics improvement of triangular dielectric resonator antenna," in 2019 International Symposium on Networks, Computers and Communications (ISNCC), Istanbul, Turkey, June 2019. 